\title{
Chemical Identification of Some Toxic Residues Bioaccumulated in Date Palm Seeds (Phoenix dactylifera)
}

\author{
Najla Bentrad 1,* iD, Rabéa Gaceb-Terrak ${ }^{1}$ \\ 1 Laboratory Research on Arid Zones, Faculty of Biological Sciences, Department of Biology and Physiology of Organisms, \\ University of Sciences and Technology Houari Boumediene (USTHB), BP 32, 16111 El-Alia, Bab Ezzouar, Algiers \\ (Algeria) \\ * Correspondence: bentrad.najla@gmail.com; nbentrad@usthb.dz;
}

Scopus Author ID 57193903517

Received: 1.06.2020; Revised: 22.06.2020; Accepted: 23.06.2020; Published: 26.06.2020

\begin{abstract}
The dangers resulting in the consumption of contaminated elements found on date fruits or seeds products after exposure to pesticides and toxic chemicals are harmful to health. In the present study, the organic extract of date palm seeds was analyzed using gas chromatography coupling-mass spectrometry (GC/MS). The results obtained show a high concentration of fatty substances such as trans-oleic acid, the most abundant unsaturated fatty acid (18.67\%) followed by monoenoic fatty acids, such as oleic acid methyl ester $(8.87 \%)$. Secondary metabolites such as phenol, 2,6-di-tert-butyl(4.97\%), beta-ionone, methyl-(1.05\%) and alkaloids such as elaeagnine (1.22\%) were measured at low concentrations. Thirteen compounds belonging to linear hydrocarbons were found at a rate of $12.08 \%$. The seeds were also exposed to pesticides such as diethyl parathion $(9.84 \%)$ and toxic residues such as thiophene,3-butyl-,1,1-dioxide (0.51\%) known to be toxic for human consumption.
\end{abstract}

Keywords: Date palm seeds; GC/MS; fatty substances; hydrocarbons; pesticide residues; hydrocarbons.

(c) 2020 by the authors. This article is an open-access article distributed under the terms and conditions of the Creative Commons Attribution (CC BY) license (https://creativecommons.org/licenses/by/4.0/).

\section{Introduction}

Particular attention must be given to the role of environmental technologies in monitoring pollutants. Their emissions into the environment, to identify the hazards of pollutants to the environment, and understand the tolerance levels of persistent pollutants in plants [1]. Actually, it is possible to use green plants as rapid biosensors for environmental identification, tracking, and the detection of the effects of pesticides, heavy metal pollutants, and other microbial contaminants [1,2]. For example, contamination of agricultural products by some pesticides residues potentially harmful compounds which could impact human health [3]. Most farms that produce vegetables and fruit are treated with more than one plant protection product or pesticides, the hazards posed by pesticides must be controlled to reduce their entry into the food chain [4,5]. These environmental problems have led to the possible use of various methods such as microextraction, associated with an analysis of micellar electrokinetic chromatography for the determination of pesticides in environmental samples and plants [6]. According to some information on exposure to environmental pollution and chemical mixtures such as toxins of chemical origin, metalloids can often be caused and accumulated during storage conditions [7], the level of toxic residues is revealed by chemical analysis of a food supplement, sometimes it shows some differences between what is indicated 
on the label and the detected values [8]. Although the rapid screening methods allow the analysis of a large number of samples, the mass spectrometry methods with multiple analytes led full quantification of the analytes [9]. Risk assessment of individual exposure to pesticides induce mood disorders, and it has been documented that exposure to organophosphate pesticides type affects neuronal regeneration, as results in the mechanism of toxicological action, may lead to the recorded increase in the risk of developing anxiety and depression following occupational exposure to organophosphate pesticides [10]. Therefore, it is important to demand more stringent regulations to set the same quality standards because of preventing the harmful effects they may have on the consumer. The world's population was currently using herbal and natural remedies for medical care [11]. The fruits and seeds of the date palm (Phoenix dactylifera) have beneficial properties that can serve as a source of nutraceuticals, dietary and phytotherapeutic purposes, fruits and seeds are rich in alkaloids, proteins, carbohydrates, fatty acids, carotenoids, vitamins, polyphenols, flavonoids, and tannins, as well as various types of nutrients such as potassium, calcium, magnesium and phosphorus and various type of fatty acids [12-14]. The objective of this study is to analyze the chemical composition of date palm seeds (Phoenix dactylifera), in order to determine whether these seeds contain potentially toxic elements, in this situation, the consumption of fruit will have a direct effect on human health.

\section{Materials and Methods}

\subsection{Plant Material.}

The date fruit of Deglet Nour cultivars (AC) was harvested in autumn 2013, from Tozeur (Tunisia) $33^{\circ} 55^{\prime} 10^{\prime \prime} \mathrm{N}, 8^{\circ} 08^{\prime \prime} \mathrm{E}$, the fruits were marketed for human consumption. The seeds were reduced completely fine powder by an electric grinder (type KSW $445 \mathrm{CB}$ ) and placed in a hermetically sealed bottle.

\subsection{Detail on the extraction of the organic fraction.}

The organic extracts from date seeds were obtained according to protocol optimized by Lebreton and co-workers [15]; in the first order, acid hydrolysis was performed on 20g dry plant material blinded with $80 \mathrm{~mL}$ of hydrochloric acid $(2 \mathrm{~N} \mathrm{HCl})$. After that, the mixture prepared into the Erlenmeyer flask was boiled in a water bath at $100{ }^{\circ} \mathrm{C}$ for 40 minutes. To extract chemical compounds, the acid mixture is separated twice into two fractions with diethyl ether $(60-60 \mathrm{~mL})$. The colors of fractions obtained are light yellow corresponding to organic fraction, for the first time, it dissolved in methanol and then evaporation host. For chromatographic analysis, the chemical compounds are dissolved in hexane. However, the orange-red faction was eliminated.

\subsection{GC/MS profiling of chemical composition of seeds and pollen extracts.}

The chemical analysis of the organic extract was performed by GC/MS using an HP 6800 chromatograph (Agilent Technologies) coupled to a mass spectrometer HP 5973 MSD equipped with a capillary column HP-5MS (5\% phenyl and 95\% dimethyl polysiloxane) with dimensions of $30 \mathrm{~m} \times 0.25 \mathrm{~mm} \times 0.25$ microns. The temperature in the column was programmed at $60{ }^{\circ} \mathrm{C}$ to $290{ }^{\circ} \mathrm{C}$ at $6{ }^{\circ} \mathrm{C}$. $\mathrm{min}^{-1}$ and then held isothermal for $5 \mathrm{~min}$. The gas carrier is helium and flow at a rate of $0.8 \mathrm{~mL} \cdot \mathrm{min}^{-1}$. The injector temperature was at $250{ }^{\circ} \mathrm{C}$, 
the volume of extract injected is $1 \mu \mathrm{l}$ in split mode (20:1), and the solvent is hexane (within 4 min). The interface temperature was $280{ }^{\circ} \mathrm{C}$, and of ionization source was $230{ }^{\circ} \mathrm{C}$ and the ionization was effected by electron impact (EI) with a potential of $70 \mathrm{eV}$. The analyzer was a quadrupole $\left(150{ }^{\circ} \mathrm{C}\right)$. The mass spectrum is recorded using a mass detector scan mode (34-550 $\mathrm{amu}$ ). The GC/MS can detect compounds according to their elution order and identify by comparing their mass spectrum and retention time (RT) with those of Wiley databases NIST 7 and 02 . The content of chemicals constituents is expressed by percentages peak-area (Area \%). The compounds selected for identification are those with a recognition rate higher than $30 \%$

\section{Results and Discussion}

The chemical composition of organic extract gives quantitative levels of the content, which is expressed by the distribution area in \% (Figure 1). Various compounds were found well as fatty acid, and ester bonds of fatty acids were formed under transesterification into methanol, other fatty compounds, aromatic substances, alkaloids, aliphatic hydrocarbons that were found with very low content such as toxic compounds, and pesticide residues.

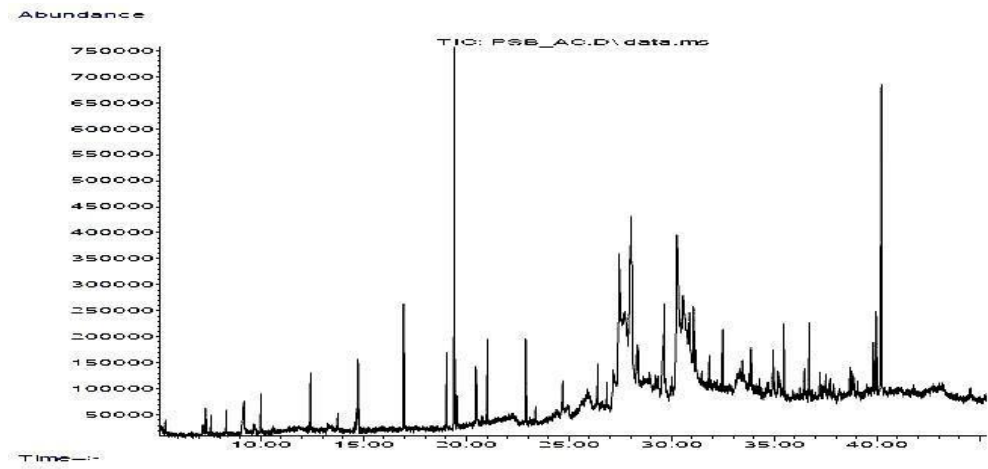

Figure 1. Chromatogram of the organic extract of date seed obtained by GC/MS analyses.

\subsection{Fatty acids.}

The analyzed date palm seeds extracts (AC) contain methyl esters of saturated and unsaturated fatty acids (\%)(Table 1 ). In general, the presence of a double bond reduces a fatty acid melting point, increases its oxidation susceptibility, and contributes to the existence of several geometric and positional isomers for the same length of the carbon chain, such as transoleic acid the most abundant unsaturated fatty acid (18.67\%) (Figure 2), followed by monoenoic fatty acids, such as oleic acid methyl ester (8.87\%). According to previous works, we can see that the soxhlet extraction of date palm seeds gives better extraction of unsaturated fatty acids compared to other fatty derivatives [16]. A study was performed to assess the chemical composition and the physicochemical properties of the seed oils from 6 varieties of date palm (Ph. dactylifera), the average free content of fatty acids was $0.5 \%$. The seed oil also had a mean content tocol and its related compounds ( 2 tocopherols and 2 tocotrienols) of 70.75 $\mathrm{mg} / 100 \mathrm{~g}$ [17]. Our results corroborate with those found by Besbes et al. [18] with a concentration in oleic acid at 39.17\%. Nehdi et al. [19] report that the major fatty acid found in the seeds of Ph. Canariensis is oleic (50\%). The seeds of some Arecaceae such as Phoenix canariensis Hort. [19] and Washingtonia filifera (Linden ex André) H. Wendl. [20] constantly contains oleic acid (40.60\%); it is the most abundant compound compared to other fatty acids. Also, the major fatty acid that is containing in seeds of Ph. Theophrasti Gr. [21] is an oleic acid type $(17.88 \%)$. A study has shown that oleic acid is the precursor of cis-vaccenic acid. The 
conversion of cis-vaccenic acid from oleic acid is considered to be a secondary isomerization pathway revealed in many fruits [22]. The coconuts (Cocos nucifera), African oil palm (Elaeis guineensis) and date palm (Phoenix dactylifera) are the Arecaceae family's three main cultivated species, for which genome sequences have been recently made available to clarify the similarities and differences in lipid metabolism between the three main species of Arecaceae [23], it provides valuable details for physiological studies; the genes involved in transforming pyruvate into fatty acid and many of the lipid-related genes were strongly homologous and conserved in homologous segments for the selection of fatty acid composition and oil content in these cultures between the three species [23].

Table 1. Chemical composition of the organic extract of Deglet Nour seeds cultivars analyzed by GC/MS.

\begin{tabular}{|c|c|c|c|c|c|c|}
\hline $\mathbf{N}^{\circ}$ & Compound & IUPAC name / Synonyme & $\begin{array}{l}\text { RT } \\
(\min )\end{array}$ & $\begin{array}{l}\text { Area } \\
\%\end{array}$ & $\begin{array}{l}\text { Molecular } \\
\text { Weight }\end{array}$ & $\begin{array}{l}\text { Molecular } \\
\text { Formula }\end{array}$ \\
\hline $\mathbf{1}$ & $\begin{array}{l}\text { Pentanoic acid, 4-oxo-, } \\
\text { methyl ester }\end{array}$ & Levulinic acid, methyl ester & 7.324 & 0,41 & 130,1418 & $\mathrm{C}_{6} \mathrm{H}_{10} \mathrm{O}_{3}$ \\
\hline 2 & Decane & n-Decane & 7.577 & 0,25 & 142,28 & $\mathrm{C}_{10} \mathrm{H}_{22}$ \\
\hline 3 & NI & & 8.313 & 0,310 & & \\
\hline 4 & Pentanoic acid, 4-oxo- & $\begin{array}{l}\text { Levulinic acid / Laevulinic } \\
\text { acid }\end{array}$ & 9.178 & 1,03 & 116,11 & $\mathrm{C}_{5} \mathrm{H}_{8} \mathrm{O}_{3}$ \\
\hline 5 & Undecane & n-Undecane & 10.002 & 0,45 & 156,31 & $\mathrm{C}_{11} \mathrm{H}_{24}$ \\
\hline 6 & Dodecane & n-Dodecane & 12.420 & 0,69 & 170,33 & $\mathrm{C}_{12} \mathrm{H}_{26}$ \\
\hline 7 & $\begin{array}{l}\text { Pentanoic acid, 4-oxo-, } \\
\text { butyl ester }\end{array}$ & Levulinic acid, butyl ester & 13.744 & 0,21 & 172,22 & $\mathrm{C}_{9} \mathrm{H}_{16} \mathrm{O}_{3}$ \\
\hline 8 & Tridecane & n-Tridecane & 14.745 & 1,21 & 184,37 & $\mathrm{C}_{13} \mathrm{H}_{28}$ \\
\hline 9 & Tetradecane & n-Tetradecane & 16.952 & 1,55 & 198,38 & $\mathrm{C}_{14} \mathrm{H}_{30}$ \\
\hline $\mathbf{1 0}$ & Pentadecane & n-Pentadécane & 19.047 & 0,94 & 212,41 & $\mathrm{C}_{15} \mathrm{H}_{32}$ \\
\hline 11 & $\begin{array}{ll}\text { Phenol, } & \text { 2,6-bis(1,1- } \\
\text { dimethylethyl) } & \text {-4-methyl- }\end{array}$ & Phenol, 2,6-di-tert-butyl- & 19.424 & 4,97 & 206,32 & $\mathrm{C}_{14} \mathrm{H}_{22} \mathrm{O}$ \\
\hline 12 & $\begin{array}{l}\text { Dodecanoic acid, methyl } \\
\text { ester }\end{array}$ & Lauric acid, methyl ester & 19.553 & 0,47 & 214,34 & $\mathrm{C}_{13} \mathrm{H}_{26} \mathrm{O}_{2}$ \\
\hline 13 & $\begin{array}{l}\text { 1-Methyl-2-trifluoroethyl- } \\
1,2,3,4- \\
\text { tetrahydro-.beta.-carboline }\end{array}$ & Elaeagnine & 20.483 & 1,22 & 186,25 & $\mathrm{C}_{12} \mathrm{H}_{14} \mathrm{~N}_{2}$ \\
\hline 14 & Hexadecane & n-Hexadecane & 21.024 & 1,06 & 226,44 & $\mathrm{C}_{16} \mathrm{H}_{34}$ \\
\hline 15 & Heptadecane & n-Heptadecane & 22.908 & 1,04 & 240,48 & $\mathrm{C}_{17} \mathrm{H}_{36}$ \\
\hline 16 & $\begin{array}{l}\text { Tetradecanoic acid, methyl } \\
\text { ester }\end{array}$ & Myristic acid, methyl ester & 23.378 & 0,19 & 242,39 & $\mathrm{C}_{15} \mathrm{H}_{30} \mathrm{O}_{2}$ \\
\hline 17 & Octadecane & n-Octadecane & 24.691 & 0,53 & 254,49 & $\mathrm{C}_{18} \mathrm{H}_{38}$ \\
\hline 18 & NI & & 25.615 & 0,95 & & \\
\hline 19 & $\mathrm{NI}$ & & 25.909 & 1,89 & & \\
\hline 20 & Nonadecane & n-Nonadécane & 26.397 & 0,76 & 268,52 & $\mathrm{C}_{19} \mathrm{H}_{40}$ \\
\hline 21 & $\begin{array}{l}\text { Hexadecanoic acid, methyl } \\
\text { ester }\end{array}$ & Palmitic acid, methyl ester & 26.845 & 0,40 & 270,45 & $\mathrm{C}_{17} \mathrm{H}_{34} \mathrm{O}_{2}$ \\
\hline 22 & Tertradecanol & Tetradecan-1-ol & 27.168 & 1.89 & 214.39 & $\mathrm{C}_{14} \mathrm{H}_{30} \mathrm{O}$ \\
\hline 23 & Hexadecanoic acid & Palmitic acid / Cetylic acid & 27.457 & 7,85 & 256,42 & $\mathrm{C}_{16} \mathrm{H}_{32} \mathrm{O}_{2}$ \\
\hline 24 & Hexadecanoic acid & Palmitic acid / Cetylic acid & 27.721 & 7,11 & 256,424 & $\mathrm{C}_{16} \mathrm{H}_{32} \mathrm{O}_{2}$ \\
\hline 25 & $\begin{array}{l}\text { Phosphorothioic acid, O,O- } \\
\text { diethylO-(4-nitrophenyl) } \\
\text { ester }\end{array}$ & parathion & 28.022 & 9,84 & 263,20 & $\begin{array}{l}\mathrm{C}_{10} \mathrm{H}_{14} \mathrm{NO}_{5} \mathrm{P} \\
\mathrm{S}\end{array}$ \\
\hline 26 & Octadecanal & Octadecanaldehyde & 28.345 & 1,59 & 268,49 & $\mathrm{C}_{18} \mathrm{H}_{36} \mathrm{O}$ \\
\hline 27 & $\begin{array}{l}\text { 9-Octadecenoic acid (Z)-, } \\
\text { methyl ester }\end{array}$ & Oleic acid, methyl ester & 29.628 & 2,82 & 296,48 & $\mathrm{C}_{19} \mathrm{H}_{36} \mathrm{O}_{2}$ \\
\hline 28 & $\begin{array}{l}\text { Cyclohexane, 1-(1,5- } \\
\text { dimethylhexyl)-4-(4- } \\
\text { methylpentyl)- }\end{array}$ & $\begin{array}{l}\text { 1-(6-methylheptan-2-yl)-4- } \\
\text { (4- } \\
\text { methylpentyl)cyclohexane }\end{array}$ & 30.011 & 0,69 & 280.53 & $\mathrm{C}_{20} \mathrm{H}_{40}$ \\
\hline 29 & 9-Octadecenoic acid, (E)- & $\begin{array}{l}\text { Elaidic acid / trans-Oleic } \\
\text { acid }\end{array}$ & 30.264 & 9,37 & 282,46136 & $\mathrm{C}_{18} \mathrm{H}_{34} \mathrm{O}_{2}$ \\
\hline 30 & 9-Octadecenoic acid, (E)- & $\begin{array}{l}\text { Elaidic acid / trans-Oleic } \\
\text { acid }\end{array}$ & 30.558 & 9,30 & 282,46 & $\mathrm{C}_{18} \mathrm{H}_{34} \mathrm{O}_{2}$ \\
\hline 31 & 9-Octadecenoic acid (Z)- & Oleic acid, methyl ester & 30.864 & 3,31 & 296,48 & $\mathrm{C}_{19} \mathrm{H}_{36} \mathrm{O}_{2}$ \\
\hline 32 & Docosane & n-Docosane & 31.070 & 1,90 & 310,60 & $\mathrm{C}_{22} \mathrm{H}_{46}$ \\
\hline 33 & 9-Octadecenoic acid (Z)- & Oleic acid, methyl ester & 31.176 & 2,10 & 296,48 & $\mathrm{C}_{19} \mathrm{H}_{36} \mathrm{O}_{2}$ \\
\hline
\end{tabular}




\begin{tabular}{|c|c|c|c|c|c|c|}
\hline $\mathbf{N}^{\circ}$ & Compound & IUPAC name / Synonyme & $\begin{array}{l}\text { RT } \\
(\mathbf{m i n})\end{array}$ & $\begin{array}{l}\text { Area } \\
\%\end{array}$ & $\begin{array}{l}\text { Molecular } \\
\text { Weight }\end{array}$ & $\begin{array}{l}\text { Molecular } \\
\text { Formula }\end{array}$ \\
\hline 34 & $\begin{array}{l}\text { 3- } \\
\text { Heptafluorobutyroxypenta } \\
\text { decane }\end{array}$ & $\begin{array}{l}\text { 1-Ethyltridecyl } \\
2,2,3,3,4,4,4- \\
\text { heptafluorobutanoate }\end{array}$ & 31.829 & 0.72 & 424.4 & $\mathrm{C}_{19} \mathrm{H}_{31} \mathrm{~F}_{7} \mathrm{O} 2$ \\
\hline 35 & Tricosane & n-Tricosane & 32.488 & 0,92 & 324,62 & $\mathrm{C}_{23} \mathrm{H}_{48}$ \\
\hline 36 & 9-Octadecenoic acid (Z)- & Oleic acid, methyl ester & 33.189 & 0,64 & 296,48 & $\mathrm{C}_{19} \mathrm{H}_{36} \mathrm{O}_{2}$ \\
\hline 37 & $\begin{array}{l}\text { cis-2,3- } \\
\text { Epoxycyclohexane-1- } \\
\text { methanol }\end{array}$ & $\begin{array}{l}{[(1 \mathrm{R}, 6 \mathrm{~S})-7-} \\
\text { oxabicyclo[4.1.0]heptan- } \\
5 \text {-yl]methanol }\end{array}$ & 33.318 & 0,96 & 128,16 & $\mathrm{C}_{7} \mathrm{H}_{12} \mathrm{O}_{2}$ \\
\hline 38 & 9-Octadecenamide, $(\mathrm{Z})$ - & Oleamide; Oleic acid amide & 33.442 & 0,73 & 281,47 & $\mathrm{C}_{18} \mathrm{H}_{35} \mathrm{NO}$ \\
\hline 39 & Hexadecenoic acid, Z-11- & Z-11-Hexadecenoic acid & 33.771 & 0,38 & 254,40 & $\mathrm{C}_{16} \mathrm{H}_{30} \mathrm{O}_{2}$ \\
\hline 40 & Dotriacontane & n-Dotriacontane & 33.866 & 0,78 & 450,86 & $\mathrm{C}_{32} \mathrm{H}_{66}$ \\
\hline 41 & $\begin{array}{l}\text { 9-Octadecenoic acid (Z)-, } \\
\text { 2,3- } \\
\text { dihydroxypropyl ester }\end{array}$ & Glycerol $\alpha$-monooleate & 34.289 & 0,22 & 356,53 & $\mathrm{C}_{21} \mathrm{H}_{40} \mathrm{O}_{4}$ \\
\hline 42 & $\mathrm{NI}$ & & 34.678 & 0,38 & & \\
\hline 43 & 1H-Indole, 5-methyl- & $\begin{array}{l}\text { 1H-Indole, } \\
\text { monohydrate }\end{array}$ & 34.942 & 1,10 & 149.19 & $\mathrm{C}_{9} \mathrm{H}_{11} \mathrm{NO}$ \\
\hline 44 & $\begin{array}{l}\text { Silane, } \\
\text { methylenebis[dimethyl- }\end{array}$ & $\begin{array}{l}\text { (E)-1-(2,6,6- } \\
\text { trimethylcyclohexen-1- } \\
\text { yl)pent-1-en-3-one }\end{array}$ & 35.184 & 0,47 & 132.35 & $\mathrm{C}_{5} \mathrm{H}_{16} \mathrm{Si}_{2}$ \\
\hline 45 & 1,9-Tetradecadiene & (9Z)-tetradeca-1,9-diene & 35.249 & 0,47 & 194.36 & $\mathrm{C}_{14} \mathrm{H}_{26}$ \\
\hline 46 & .beta.-Ionone, methyl- & $\begin{array}{l}\text { (1E)-1-(2,6,6-Trimethyl-1- } \\
\text { cyclohexen-1-yl)-1-penten- } \\
\text { 3-one }\end{array}$ & 35.466 & 1,05 & 206,32 & $\mathrm{C}_{14} \mathrm{H}_{22} \mathrm{O}$ \\
\hline 47 & Nonahexacontanoic acid & n-Nonahexacontansaeure & 36.467 & 0,78 & 999.8 & $\mathrm{C}_{69} \mathrm{H}_{138} \mathrm{O}_{2}$ \\
\hline 48 & $\begin{array}{l}\text { 2-methyl 3-(4-(1'- } \\
\text { methylethyl)-phenyl) } \\
\text { propanal }\end{array}$ & $\begin{array}{l}\text { 3-(4-Isopropylphenyl)-2- } \\
\text { methylpropionaldehyde }\end{array}$ & 36.690 & 1,10 & 190,28 & $\mathrm{C}_{13} \mathrm{H}_{18} \mathrm{O}$ \\
\hline 49 & $\begin{array}{l}\text { 3-(2,2-dideuterobutyl)- } \\
\text { thiophene-1,1-dioxide }\end{array}$ & $\begin{array}{l}\text { Thiophene, 3-butyl-, 1,1- } \\
\text { dioxide }\end{array}$ & 37.208 & 0,51 & 172,24 & $\mathrm{C}_{8} \mathrm{H}_{12} \mathrm{O}_{2} \mathrm{~S}$ \\
\hline 50 & 1H-Indole, 7-methyl- & 7-methyl-1H-indole & 37.497 & 0,49 & 131.17 & $\mathrm{C}_{9} \mathrm{H}_{9} \mathrm{~N}$ \\
\hline 51 & Thiophene, 2-hexyl- & 2-hexylthiophene & 37.714 & 0,75 & 168.29 & $\mathrm{C}_{10} \mathrm{H}_{16} \mathrm{~S}$ \\
\hline 52 & .beta.-Ionone, methyl- & $\begin{array}{l}\text { (E)-1-(2,6,6- } \\
\text { trimethylcyclohexen-1- } \\
\text { yl)pent-1-en-3-one }\end{array}$ & 37.897 & 0,33 & 206,32 & $\mathrm{C}_{14} \mathrm{H}_{22} \mathrm{O}$ \\
\hline 53 & $\mathrm{NI}$ & & 38.679 & 0,64 & & \\
\hline 54 & $\mathrm{NI}$ & & 38.780 & 0,48 & & \\
\hline 55 & Dodecane, 1,2-dibromo- & 1,2-Dibromododecane & 38.862 & 0,51 & 300,07 & $\mathrm{C}_{10} \mathrm{H}_{20} \mathrm{Br}_{2}$ \\
\hline 56 & $\begin{array}{l}\text { 1H-Indene, } 2,3,3 \mathrm{a}, 4,7,7 \mathrm{a}- \\
\text { hexahydro-2,2,4,4,7,7- } \\
\text { hexamethyl- }\end{array}$ & $\begin{array}{l}(3 \mathrm{aR}, 7 \mathrm{aR})-2,2,4,4,7,7- \\
\text { hexamethyl-1,3,3a,7a- } \\
\text { tetrahydroindene }\end{array}$ & 39.056 & 0,26 & 206.37 & $\mathrm{C} 15 \mathrm{H} 26$ \\
\hline 57 & $\begin{array}{l}\text { Cyclooctene, 4-methylene- } \\
\text { 6-(1-propenylidene)- }\end{array}$ & $\begin{array}{l}\text { (1Z)-4-methylidene-6- } \\
\text { prop-1- } \\
\text { enylidenecyclooctene }\end{array}$ & 39.821 & 0,87 & 160.25 & $\mathrm{C}_{12} \mathrm{H}_{16}$ \\
\hline 58 & $\begin{array}{l}\text { Cholesta-4,6-dien-3-ol, } \\
\text { benzoate, } \\
\text { (3.beta.)- }\end{array}$ & $\begin{array}{l}\text { [10,13-dimethyl-17-(6- } \\
\text { methylheptan-2-yl)- } \\
\text { 2,3,8,9,11,12,14,15,16,17- } \\
\text { decahydro-1H- } \\
\text { cyclopenta[a]phenanthren- } \\
\text { 3-yl] benzoate }\end{array}$ & 39.968 & 1,30 & 384,63 & $\mathrm{C}_{27} \mathrm{H}_{44} \mathrm{O}$ \\
\hline 59 & $\begin{array}{l}\text { Cholest-5-ene, 3-bromo-, } \\
\text { (3.beta.)-, }\end{array}$ & $\begin{array}{l}\text { (3S,8S,9S,10R,13R,14S,17 } \\
\text { R)-3-bromo-10,13- } \\
\text { dimethyl-17-[(2R)-6- } \\
\text { methylheptan-2-yl]- } \\
\text { 2,3,4,7,8,9,11,12,14,15,16, } \\
\text { 17-dodecahydro-1H- } \\
\text { cyclopenta[a]phenanthrene }\end{array}$ & 40.210 & 4,84 & 449,55 & $\mathrm{C}_{27} \mathrm{H}_{45} \mathrm{Br}$ \\
\hline \multicolumn{4}{|c|}{ Number of substances detected } & \multicolumn{3}{|l|}{59} \\
\hline \multicolumn{4}{|c|}{ Total area $(\%)$} & \multicolumn{3}{|l|}{99,98} \\
\hline \multicolumn{4}{|c|}{ Number of unidentified substances (NI) } & \multicolumn{3}{|l|}{6} \\
\hline
\end{tabular}

\subsection{Other fatty compounds and phytosterols.}

Certain fatty compounds have been found in date palm seeds (Figure 2) such as $\alpha$ monooleate glycerol $(0.22 \%)$, it is an amphiphilic lipid and biodegradable compound, with 
many useful applications [24-26]. The $\alpha$-monooleate glycerol is a friction agent commonly used in the lubricant industry. It is thought to have lubricated metallic surfaces by its hydrolyzing to oleic acid, glycerol, and calcium oleate $\left(\mathrm{CaOl}_{2}\right)$ [27]. Also, the oleamide (cis9,10 -octadecenoamide) is found in date palm seeds at $0.73 \%$. It is a fatty acid amide discovered in cerebrospinal fluid in sleep-deprived animals [28], has a variety of effects that impart as signaling molecules potential in some biological activities [28,29].
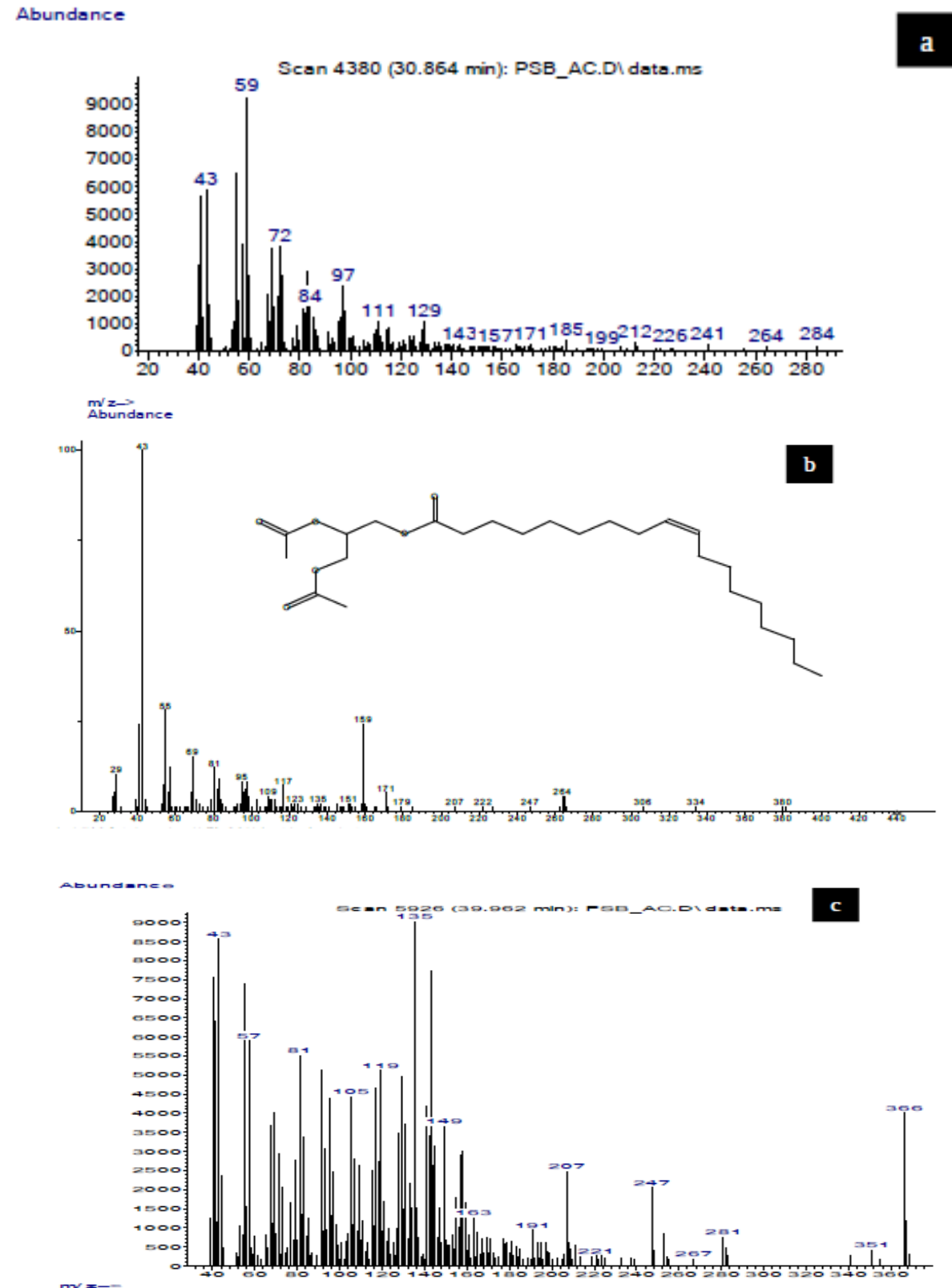

Figure 2. Mass spectrum of principal fatty compounds found in date palm seeds: a-oleamide; b- glycerol $\alpha$-monooleate; c- 4,6-cholestadien-3 $\beta$-ol.

Indeed, 4,6-cholestadien-3 $\beta$-ol (1.3\%) identified in date palm seeds was also found in some crude extracts from Brazilian and Spanish marine species (Condrosia reniformes, Tethya rubra, and Tethya ignis) [30]. However, the $3 \beta$-bromo-5-cholestene (4.84\%) was isolated (Figure 3) for the first time from the date seeds. 


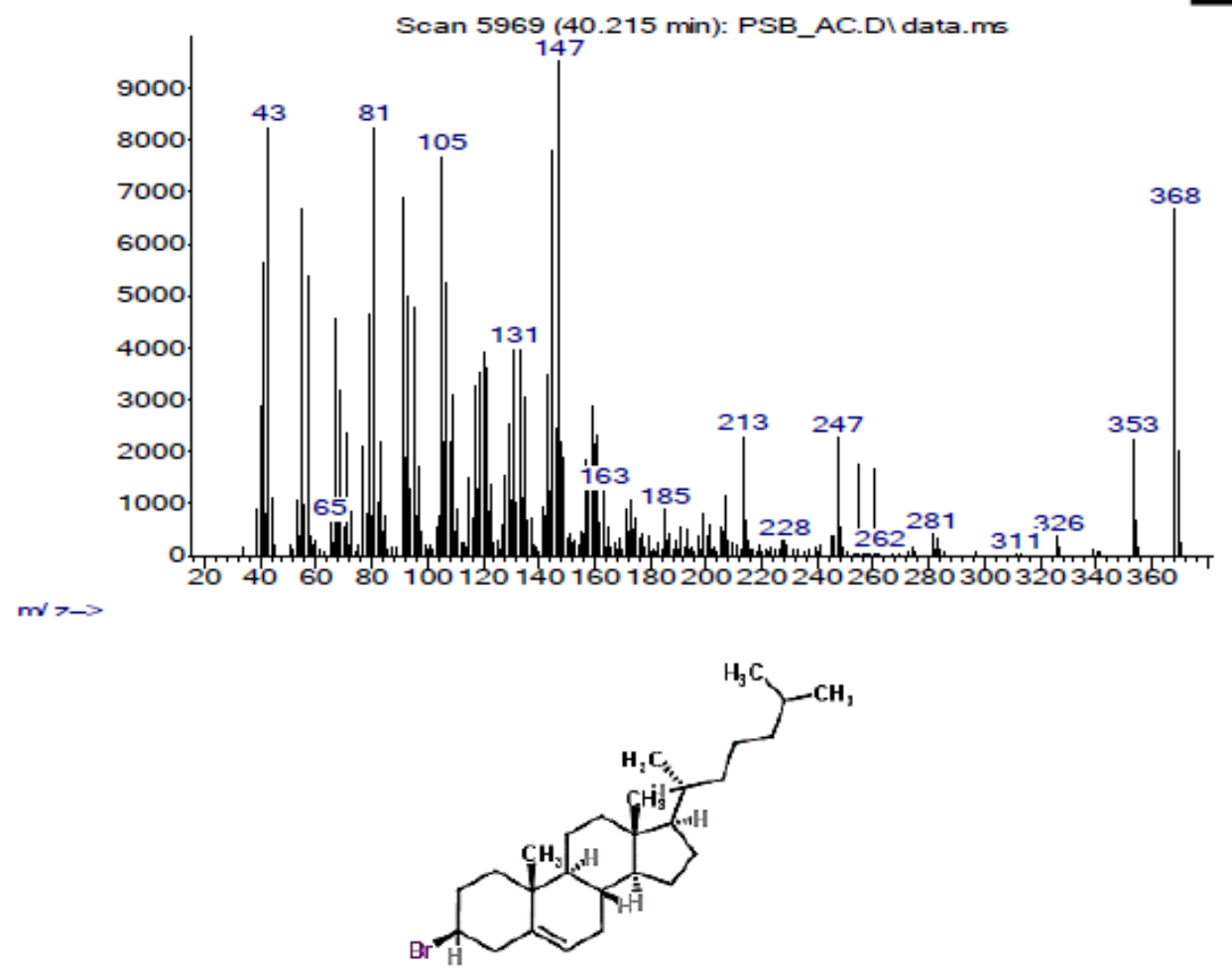

Figure 3. Mass spectrum, cholest-5-ene 3-bromo-, (3.beta.)-( a) and its structural formula (b) found in seed date.

\subsection{Aromatics and secondary metabolites.}

The analyzes using CG/SM revealed the presence of secondary metabolites (Figure 4) such as phenol 2,6-di-tert-butyl-(4.97\%); 3-(4-isopropylphenyl)-2-methylpropionaldehyde $(1.1 \%)$ a cyclamen aldehyde a colorless liquid may turn to pale yellow. It is considered a strong floral aroma [31].

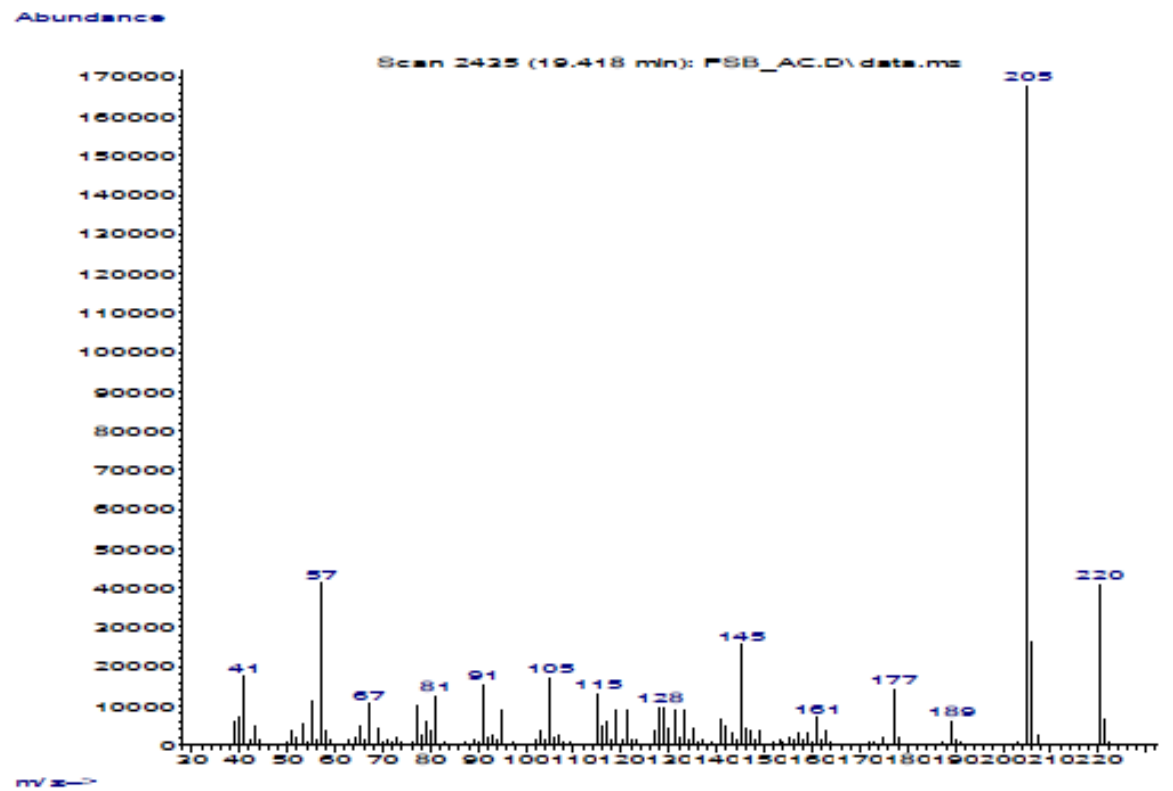

Figure 4. Mass spectrum of phenol, 2,6-bis (1,1-dimethylethyl) -4-methyl- found in date palm seeds. 
A terpene molecule beta-ionone,methyl-(1.05\%) has also been identified, and it is used in many perfumes. According to some studies, the average skin content resulting from the use of isomethyl-b-ionone and its derivatives in fine fragrance formulae would be used at a rate of $1.18 \%[32,33]$ in the final product. However, alkaloids elaeagnine $(1.22 \%)$ is found in date palm seeds for the first time.

\subsection{Hydrocarbons.}

Hydrocarbons (Table 1) are quite found in date palm seeds. They are mainly represented by saturated linear alkanes such as n-decane, n-undecane, n-dodecane, n-tridecane, n-tetradecane, n-pentadecane, n-hexadecane, n-heptadecane, n-octadecane, n-nonadecane, ndocosane, $n$-tricosane and $n$-dotriacontane with a rate of $12.08 \%$. The hydrocarbons can be found in food due to contamination from various origins [32-37], they have been found in many food products, including edible vegetable oils which, due to their lipophilic nature, are easily contaminated and widely distributed in the environment and known to be carcinogens [33]. Therefore environmental management requires an understanding of the bioaccumulation and the using of various analytic methods to estimate the rate of hydrocarbon in plants [33,34]. Two main routes of hydrocarbon pollution in vegetable oils have been suggested [35,36]: interaction with the contaminated atmosphere and the method of drying the raw material by combustion fumes and natural gas. Air pollution from dust and particles containing large quantities of hydrocarbons can contaminate plants by air emissions during the growth vegetation phase [32]. It can be found in diesel engine exhaust particles [36]. This surface contamination can be passed to the final product or during the food packaging process [38].

\subsection{Toxic chemical compounds and pesticide residues.}

Concentrations of pollutants and potentially toxic elements in seeds are represented in low contents, such as thiophene, 3-butyl-,1,1-dioxide $(0.51 \%)$. Also, it has been revealed the presence of residues of pesticides such as diethyl parathion $(9.84 \%)$ (Figure 5).

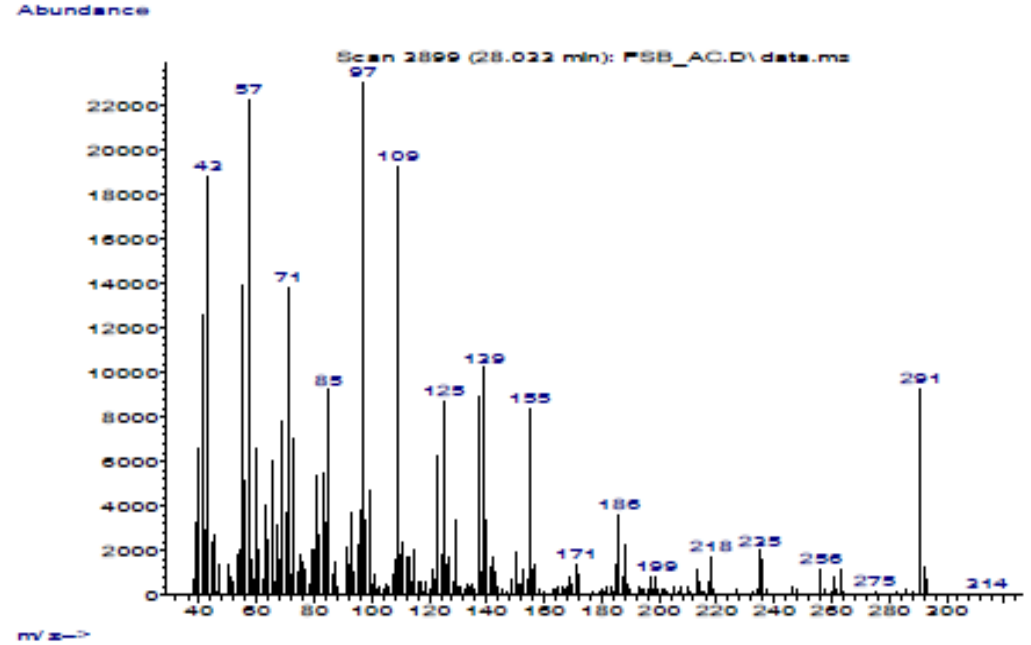

Figure 5. Mass spectrum of phosphorothioic acid, O,O-diethylO-(4-nitrophenyl) ester found in date palm seeds.

Due to its introduction into the general environment, pesticide residues are found in food and are toxic to insects [39]. Indeed, organophosphate pesticides are often used to control insect perforations that can pose a risk to bees [40]. The latest studies on the risks associated 
with organic phosphate exposure have been reported [41,42] pesticides have a wide range of physicochemical properties. However, they are particularly difficult to extract in order to facilitate their analysis from various vegetation and environment sources in different samples [43]. So, we can deduce that organic contaminants can enter the vegetation by the absorption process from the atmospheric and environmental contaminants, and in general, it depends on the type of toxic compounds [44,45]. At the same time, co-extraction of fats and other components of the matrix is very difficult to avoid, the isolation of pesticides and other chemical pollutants from high-fat food samples before subsequent analytical measures is still a challenging problem to which a great deal of effort has been made in the development of methods [46], especially given that the majority of pesticides are lipophilic compounds [47].

\section{Conclusions}

Plants are constantly exposed to a variety of disturbances caused by air and soil pollution, drought, deficiency, or excess of nutrients. However, over a long period, only certain plants react quickly to environmental stress. The risks resulting from the exposure to toxic chemicals elements of the fruits of the date palm (Phoenix daclylifera) were analyzed using the gas chromatography coupling mass spectrometry (GC/MS). The concentrations of various class fatty substances were detected, secondary metabolites including phenol, 2,6-di-tert-butyl, and alkaloids such as eleagnine were also identified at low concentration. However, date palm seeds have been exposed to pesticides, such as thiophene, 3-butyl, and 1,1-dioxide, and about thirteen linear hydrocarbons compounds have also been found in date palm seed. Based on this study, human consumption of date palm seeds may have harmful effects; however, it can be used as rapid biosensors for the detection of pesticide, hydrocarbons, and its pollutant effects in surface water or a contaminated environment.

\section{Funding}

This research received no external funding.

\section{Acknowledgments}

We want to thank the General Directorate of Scientific Research and Technological Development (DGRSDT) in Algeria for their financial support.

\section{Conflicts of Interest}

The authors declare no conflict of interest.

\section{References}

1. Volkov, A.G.; Ranatunga, D.R.A. Plants as Environmental Biosensors. Plant Signaling \& Behavior 2006, 1, 105-115, https://doi.org/10.4161/psb.1.3.3000.

2. Paz-Alberto, A.M.; Sigua, G.C. Phytoremediation: A Green Technology to Remove Environmental Pollutants. American Journal of Climate Change 2013, 2, 71-86, https://doi.org/10.4236/ajcc.2013.21008.

3. Calatayud-Vernich, P.; Calatayud, F.; Simó, E.; Picó, Y. Pesticide residues in honey bees, pollen and beeswax: Assessing beehive exposure. Environmental Pollution 2018, 241, 106-114, https://doi.org/10.1016/j.envpol.2018.05.062.

4. Ji, F.; Zhao, L.; Yan, W.; Feng, Q.; Lin, J.-M. Determination of triazine herbicides in fruits and vegetables using dispersive solid-phase extraction coupled with LC-MS. Journal of Separation Science 2008, 31, 961968, https://doi.org/10.1002/jssc.200700610. 
5. Fosu, P.O.; Donkor, A.; Ziwu, C.; Dubey, B.; Kingsford-Adaboh, R.; Asante, I.; Nyarko, S.; Tawiah, R.; Nazzah, N. Surveillance of pesticide residues in fruits and vegetables from Accra Metropolis markets, Ghana, 2010-2012: a case study in Sub-Saharan Africa. Environmental Science and Pollution Research 2017, 24, 17187-17205, https://doi.org/10.1007/s11356-017-9287-8.

6. Yang, Q.; Chen, B.; He, M.; Hu, B. Sensitive determination of seven triazine herbicide in honey, tomato and environmental water samples by hollow fiber based liquid-liquid-liquid microextraction combined with sweeping micellar electrokinetic capillary chromatography. Talanta 2018, 186, 88-96, https://doi.org/10.1016/j.talanta.2018.04.012.

7. Samuel-Nakamura, C.; Hodge, F.S. Occurrence and Risk of Metal(loid)s in Thelesperma megapotamicum Tea Plant. 2020, 9, https://doi.org/10.3390/plants9010021.

8. Figueiredo, A.; Costa, I.M.; Fernandes, T.A.; Gonçalves, L.L.; Brito, J. Food Supplements for Weight Loss: Risk Assessment of Selected Impurities. Nutrients 2020, 12, https://doi.org/10.3390/nu12040954.

9. Krska, R.; Becalski, A.; Braekevelt, E.; Koerner, T.; Cao, X.-L.; Dabeka, R.; Godefroy, S.; Lau, B.; Moisey, J.; Rawn, D.F.K.; Scott, P.M.; Wang, Z.; Forsyth, D. Challenges and trends in the determination of selected chemical contaminants and allergens in food. Analytical and Bioanalytical Chemistry 2012, 402, 139-162, https://doi.org/10.1007/s00216-011-5237-3.

10. Judge, S.J.; Savy, C.Y.; Campbell, M.; Dodds, R.; Gomes, L.K.; Laws, G.; Watson, A.; Blain, P.G.; Morris, C.M.; Gartside, S.E. Mechanism for the acute effects of organophosphate pesticides on the adult 5-HT system. Chemico-Biological Interactions 2016, 245, 82-89, https://doi.org/10.1016/j.cbi.2015.12.014.

11. Almahasheer, H. Nutrition in Herbal Plants Used in Saudi Arabia. Scientifica 2020, https://doi.org/10.1155/2020/6825074.

12. Qadir, A.; Shakeel, F.; Ali, A.; Faiyazuddin, M. Phytotherapeutic potential and pharmaceutical impact of Phoenix dactylifera (date palm): current research and future prospects. Journal of Food Science and Technology 2020, 57, 1191-1204, https://doi.org/10.1007/s13197-019-04096-8.

13. Hussain, M.I.; Semreen, M.H.; Shanableh, A.; Khattak, M.N.; Saadoun, I.; Ahmady, I.M.; Mousa, M.; Darwish, N.; Radeef, W.; Soliman, S.S.M. Phenolic Composition and Antimicrobial Activity of Different Emirati Date (Phoenix dactylifera L.) Pits: A Comparative Study. Plants 2019, 8, https://doi.org/10.3390/plants8110497.

14. Maqsood, S.; Adiamo, O.; Ahmad, M.; Mudgil, P. Bioactive compounds from date fruit and seed as potential nutraceutical and functional food ingredients. Food Chemistry 2020, 308, https://doi.org/10.1016/j.foodchem.2019.125522.

15. Lebreton, P.H.; Jay, M.; Voirin, B.; Bouchez, M.P. L'analyse quantitative des flavonoïdes. Chem Anal 1967, 49, 375-383.

16. Bentrad, N.; Gaceb-Terrak, R.; Rahmania, F. Identification and evaluation of antibacterial agents present in lipophilic fractions isolated from sub-products of Phoenix dactylifera. Natural Product Research 2017, 31, 2544-2548, https://doi.org/10.1080/14786419.2017.1314282.

17. Nehdi, I.A.; Sbihi, H.M.; Tan, C.P.; Rashid, U.; Al-Resayes, S.I. Chemical Composition of Date Palm (Phoenix dactylifera L.) Seed Oil from Six Saudi Arabian Cultivars. Journal of Food Science 2018, 83, 624630, https://doi.org/10.1111/1750-3841.14033.

18. Besbes, S.; Blecker, C.; Deroanne, C.; Drira, N.-E.; Attia, H. Date seeds: chemical composition and characteristic profiles of the lipid fraction. Food Chemistry 2004, 84, 577-584, https://doi.org/10.1016/S0308-8146(03)00281-4.

19. Nehdi, I.; Omri, S.; Khalil, M.I.; Al-Resayes, S.I. Characteristics and chemical composition of date palm (Phoenix canariensis) seeds and seed oil. Industrial Crops and Products 2010, 32, 360-365, https://doi.org/10.1016/j.indcrop.2010.05.016.

20. Nehdi, I.A. Characteristics and composition of Washingtonia filifera (Linden ex André) H. Wendl. seed and seed oil. Food Chemistry 2011, 126, 197-202, https://doi.org/10.1016/j.foodchem.2010.10.099.

21. Liolios, C.C.; Sotiroudis, G.T.; Chinou, I. Fatty Acids, Sterols, Phenols and Antioxidant Activity of Phoenix theophrasti Fruits Growing in Crete, Greece. Plant Foods for Human Nutrition 2009, 64, 52-61, https://doi.org/10.1007/s11130-008-0100-1.

22. Shibahara, A.; Yamamoto, K.; Takeoka, M.; Kinoshita, A.; Kajimoto, G.; Nakayama, T.; Noda, M. Novel pathways of oleic and cis-vaccenic acid biosynthesis by an enzymatic double-bond shifting reaction in higher plants. FEBS Letters 1990, 264, 228-230, https://doi.org/10.1016/0014-5793(90)80254-g.

23. Xiao, Y.; Xia, W.; Mason, A.S.; Cao, Z.; Fan, H.; Zhang, B.; Zhang, J.; Ma, Z.; Peng, M.; Huang, D. Genetic control of fatty acid composition in coconut (Cocos nucifera), African oil palm (Elaeis guineensis), and date palm (Phoenix dactylifera). Planta 2019, 249, 333-350, https://doi.org/10.1007/s00425-018-3003-x.

24. Giacomozzi, A.S.; Palla, C.A.; Carrín, M.E.; Martini, S. Physical Properties of Monoglycerides Oleogels Modified by Concentration, Cooling Rate, and High-Intensity Ultrasound. Journal of Food Science 2019, 84, 2549-2561, https://doi.org/10.1111/1750-3841.14762.

25. Giacomozzi, A.S.; Carrín, M.E.; Palla, C.A. Muffins Elaborated with Optimized Monoglycerides Oleogels: From Solid Fat Replacer Obtention to Product Quality Evaluation. Journal of Food Science 2018, 83, 15051515, https://doi.org/10.1111/1750-3841.14174. 
26. Chen, J.; Dickinson, E. Effect of monoglycerides and diglycerol-esters on viscoelasticity of heat-set whey protein emulsion gels. International Journal of Food Science \& Technology 1999, 34, 493-501, https://doi.org/10.1046/j.1365-2621.1999.00319.x.

27. Bradley-Shaw, J.L.; Camp, P.J.; Dowding, P.J.; Lewtas, K. Self-assembly and friction of glycerol monooleate and its hydrolysis products in bulk and confined non-aqueous solvents. Physical Chemistry Chemical Physics 2018, 20, 17648-17657, https://doi.org/10.1039/c8cp01785a.

28. Hiley, C.R.; Hoi, P.M. Oleamide: A Fatty Acid Amide Signaling Molecule in the Cardiovascular System? Cardiovascular Drug Reviews 2007, 25, 46-60, https://doi.org/10.1111/j.1527-3466.2007.00004.x.

29. Jug, U.; Naumoska, K.; Metličar, V.; Schink, A.; Makuc, D.; Vovk, I.; Plavec, J.; Lucas, K. Interference of oleamide with analytical and bioassay results. Scientific Reports 2020, 10, https://doi.org/10.1038/s41598020-59093-1.

30. de Paula, J.C.; Desoti, V.C.; Sampiron, E.G.; Martins, S.C.; Ueda-Nakamura, T.; Ribeiro, S.M.; Bianco, E.M.; de Oliveira Silva, S.; de Oliveira, G.G.; Nakamura, C.V. Trypanocidal activity of organic extracts from the Brazilian and Spanish marine sponges. Revista Brasileira de Farmacognosia 2015, 25, 651-656, https://doi.org/10.1016/j.bjp.2015.08.011.

31. FAO/WHO Expert Committee on Food Additives. Combined compendium of food additive specifications. All specifications monographs from the 1st to the 65th meeting (1956-2005). Last updated (Web version): August 2011. http://www.fao.org/food/food-safety-quality/scientific-advice/jecfa/jecfaflav/details/fr/c/1453/

32. Belsito, D.; Bickers, D.; Bruze, M.; Calow, P.; Greim, H.; Hanifin, J.M.; Rogers, A.E.; Saurat, J.H.; Sipes, I.G.; Tagami, H. A toxicologic and dermatologic assessment of ionones when used as fragrance ingredients. Food and Chemical Toxicology 2007, 45, S130-S167, https://doi.org/10.1016/j.fct.2007.09.067.

33. Tong, T.; Park, J.; Moon, Y.; Kang, W.; Park, T. $\alpha$-Ionone Protects Against UVB-Induced Photoaging in Human Dermal Fibroblasts. Molecules 2019, 24, https://doi.org/10.3390/molecules24091804.

34. Paris, A.; Ledauphin, J.; Poinot, P.; Gaillard, J.-L. Polycyclic aromatic hydrocarbons in fruits and vegetables: Origin, analysis, and occurrence. Environmental Pollution 2018, 234, 96-106, https://doi.org/10.1016/j.envpol.2017.11.028.

35. Lee, Y.-N.; Lee, S.; Kim, J.-S.; Kumar Patra, J.; Shin, H.-S. Chemical analysis techniques and investigation of polycyclic aromatic hydrocarbons in fruit, vegetables and meats and their products. Food Chemistry 2019, 277, 156-161, https://doi.org/10.1016/j.foodchem.2018.10.114.

36. Tao, S.; Jiao, X.C.; Chen, S.H.; Liu, W.X.; Coveney, R.M.; Zhu, L.Z.; Luo, Y.M. Accumulation and distribution of polycyclic aromatic hydrocarbons in rice (Oryza sativa). Environmental Pollution 2006, 140, 406-415, https://doi.org/10.1016/j.envpol.2005.08.004.

37. Grova, N.; Feidt, C.; Crépineau, C.; Laurent, C.; Lafargue, P.E.; Hachimi, A.; Rychen, G. Detection of Polycyclic Aromatic Hydrocarbon Levels in Milk Collected Near Potential Contamination Sources. J Agric Food Chem 2002, 50, 4640-4642, https://doi.org/doi:10.1021/jf0201071.

38. Neukom, H.P.; Grob, K.; Biedermann, M.; Noti, A. Food contamination by C20-C50 mineral paraffins from the atmosphere. Atmospheric Environment 2002, 36, 4839-4847, https://doi.org/10.1016/S13522310(02)00358-8.

39. Robidoux, P.Y.; Lopez-Gastey, J.; Choucri, A.; Sunahara, G.I. Procedure to Screen Illicit Discharge of Toxic Substances in Septic Sludge Received at a Wastewater Treatment Plant. Ecotoxicology and Environmental Safety 1998, 39, 31-40, https://doi.org/10.1006/eesa.1997.1607.

40. Biedermann, M.; Grob, K. On-line coupled high performance liquid chromatography-gas chromatography for the analysis of contamination by mineral oil. Part 2: Migration from paperboard into dry foods: Interpretation of chromatograms. Journal of Chromatography A 2012, 1255, 76-99, https://doi.org/10.1016/j.chroma.2012.05.096.

41. DiBartolomeis, M.; Kegley, S.; Mineau, P.; Radford, R.; Klein, K. An assessment of acute insecticide toxicity loading (AITL) of chemical pesticides used on agricultural land in the United States. PLOS ONE 2019, 14, https://doi.org/10.1371/journal.pone.0220029.

42. Yao, J.; Zhu, Y.C.; Adamczyk, J.; Luttrell, R. Influences of acephate and mixtures with other commonly used pesticides on honey bee (Apis mellifera) survival and detoxification enzyme activities. Comparative Biochemistry and Physiology Part C: Toxicology \& Pharmacology 2018, 209, 9-17, https://doi.org/10.1016/j.cbpc.2018.03.005.

43. Asubiojo, O.I.; Adebiyi, F.M.; Ayenimo, J.G.; Olukoko, O.O.; Oyekunle, J.A.O. Chemical analysis of tobacco cigarette for organochlorine insecticides and heavy metal composition. Toxicological \& Environmental Chemistry 2009, 91, 611-618, https://doi.org/10.1080/02772240802343123.

44. Al Naggar, Y.; Codling, G.; Giesy, J.P. Human dietary intake and hazard characterization for residues of neonicotinoides and organophosphorus pesticides in Egyptian honey. Toxicological \& Environmental Chemistry 2017, 99, 1397-1408, https://doi.org/10.1080/02772248.2017.1384828.

45. Boulanouar, S.; Mezzache, S.; Combès, A.; Pichon, V. Molecularly imprinted polymers for the determination of organophosphorus pesticides in complex samples. Talanta 2018, 176, 465-478, https://doi.org/10.1016/j.talanta.2017.08.067. 
46. Pang, N.; Wang, T.; Hu, J.; Dong, B. Field evaluation and determination of four herbicides in a wheat ecosystem by a simple and versatile QuEChERS method with liquid chromatography-tandem mass spectrometry. Toxicological \& Environmental Chemistry 2017, 99, 376-389, https://doi.org/10.1080/02772248.2016.1196209.

47. Simonich, S.L.; Hites, R.A. Organic Pollutant Accumulation in Vegetation. Environmental Science \& Technology 1995, 29, 2905-2914, https://doi.org/10.1021/es00012a004.

48. Gilbert-López, B.; García-Reyes, JF.; Molina-Díaz, A. Sample treatment and determination of pesticide residues in fatty vegetable matrices: A review. Talanta 2009, 79, 109-128. https://doi.org/10.1016/j.talanta.2009.04.022.

49. Zayats, M.F.; Leschev, S.M.; Petrashkevich, N.V.; Zayats, M.A.; Kadenczki, L.; Szitás, R.; Dobrik, H.S.; Keresztény, N. Distribution of pesticides in n-hexane/water and n-hexane/acetonitrile systems and estimation of possibilities of their extraction isolation and preconcentration from various matrices. Analytica Chimica Acta 2013, 774, 33-43, https://doi.org/10.1016/j.aca.2013.03.003. 